\title{
Theoretical Determination of Droplet Diameters and Flow Rates in Sprays and Aerosols
}

\author{
Maksim Mezhericher*1, Howard A. Stone ${ }^{1}$ \\ ${ }^{1}$ Department of Mechanical and Aerospace Engineering, Princeton University, Princeton, \\ USA \\ *Corresponding author email: maksymm@princeton.edu
}

\begin{abstract}
In our recent work we demonstrated a novel liquid atomization process generating microsprays and aerosols of submicron-diameter droplets for pure solvents, suspensions, and solutions. Our novel atomization process is based on disintegration by gas jets of thin liquid films formed as bubbles on a liquid surface. In this paper we show that the diameters and flow rates of the produced droplets are governed by the interplay of process timescales including capillary breakup, liquid viscosity, and gas jet pressure. Timescale ratios can be converted into the ratios of specific energies and into the ratios of specific energy rates provided by the gas jets and dissipated by the atomized liquid. Using those ratios, we develop new theoretical approach to determine droplet diameters and flow rates in sprays and aerosols and intoduce atomization diagrams. The comparison between theoretically predicted and measured droplet diameters and droplet flow rates for various liquids (water, gasoline, diesel and others) demonstrated good agreement for the new liquid atomization process generating micro-sprays and aerosols of submicron-diameter droplets.
\end{abstract}

\section{Keywords}

Atomization diagram, diameter of droplet, dimensionless number, flow rate.

\section{Introduction}

In our previous articles [1,2] we demonstrated a novel liquid atomization process generating micro-sprays and aerosols of submicron-diameter droplets for pure solvents, suspensions, and solutions with wide ranges of viscosity and surface tension. The process is based on gas jetting on thin liquid films formed as bubbles on a liquid surface (see Fig. 1a). The research question addressed in this study is development of a theoretical description for calculation of droplet diameters and flow rates produced by the new liquid atomization process for various liquids.

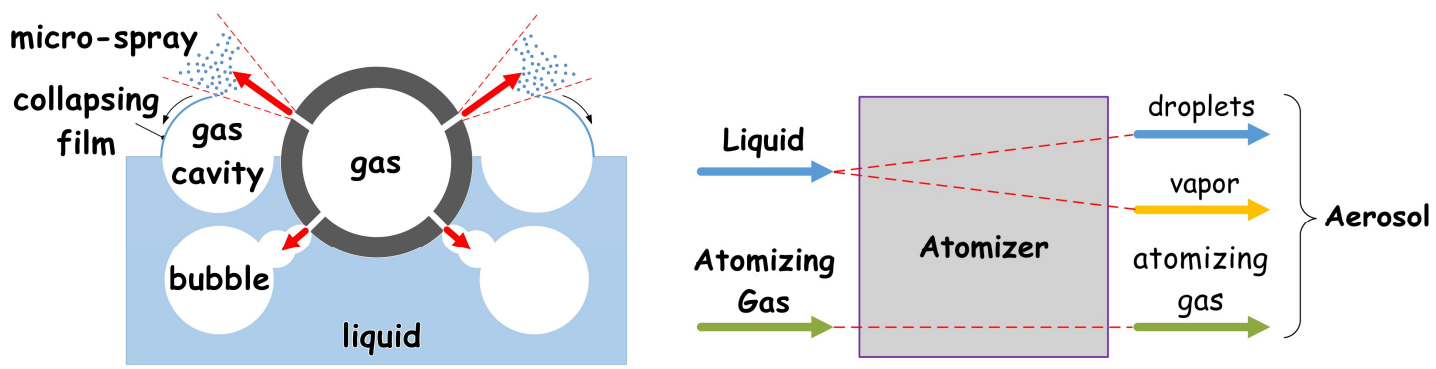

Figure 1. (a) Schematic of liquid atomization process including generation of fine droplets by gas jet disintegration of thin liquid films formed as bubbles on a liquid surface . (b) Control volume for thermodynamic analysis. 


\section{Theoretical Model}

\section{Droplet Diameters}

The developed liquid atomization process is analysed by applying the law of mass conservation and the First Law of Thermodynamics for the control volume shown in Fig. $1 \mathrm{~b}$. Assuming steady-state flow for a continuous adiabatic process at room temperature, neglecting the changes in potential energy of both fluids, evaporation and the change in liquid kinetic energy, assuming complete ideal expansion of the gas jet, and disregarding drag, we get:

$$
e_{\Delta p}=e_{c}+e_{v} \text {. }
$$

Using dimensional analysis in our previous work [1], we established that two dimensionless groups $\mathrm{La}_{d}=\rho_{l} \gamma_{1} d / \mu_{l}^{2}$ and $\mathrm{N}_{d}=\Delta p_{g j} d / \gamma_{1}$ play the central role for determining droplet diameters. Using the characteristic timescales of the liquid atomization, including the supplied energy of the gas jet $\tau_{\Delta p} \sim\left(\rho_{l} l_{c}^{2} / \Delta p_{g j}\right)^{1 / 2}$, capillary Rayleigh breakup $\tau_{c} \sim\left(\rho_{l} l_{c}^{3} / \gamma_{l}\right)^{1 / 2}$ and viscous dissipation $\tau_{v} \sim \rho_{l} l_{c}^{2} / \mu_{l}$ in the liquid, with the approximation $I_{c}=d$ for all the phenomena, the dimensionless groups can be expressed as timescale ratios:

$$
\begin{aligned}
& \mathrm{La}_{d} \sim \frac{\tau_{v}^{2}}{\tau_{c}^{2}}, \\
& \mathrm{~N}_{d} \sim \frac{\tau_{c}^{2}}{\tau_{\Delta p}^{2}} .
\end{aligned}
$$

On the other hand, the energy scales and timescales are connected, $e_{\Delta p} \sim I_{c}^{2} / \tau_{\Delta p}^{2}, e_{c} \sim I_{c}^{2} / \tau_{c}^{2}$ and $e_{v} \sim I_{c}^{2} / \tau_{v}^{2}$, and we again assume $I_{c}=d$. Therefore, from Eqs. (2) and (3) we obtain:

$$
\mathrm{La}_{d} \sim \frac{e_{c}}{e_{v}}
$$

$$
\mathrm{N}_{d} \sim \frac{e_{\Delta p}}{e_{c}}
$$

Rewriting with proportionality coefficients, we get:

$$
\begin{aligned}
& \mathrm{La}_{d}=k_{1} \frac{e_{c}}{e_{v}}, \\
& \mathrm{~N}_{d}=k_{2} \frac{e_{\Delta p}}{e_{c}} .
\end{aligned}
$$

Combining Eq. (1) with Eqs. (6), (7), we find:

$$
\mathrm{N}_{d}=k_{1}+\frac{k_{1} k_{2}}{\mathrm{La}_{d}} \text {. }
$$

The obtained Eq. (8) connects between the specific energies provided by the gas jets and dissipated by the atomized liquid in nondimensional form and determines the diameters of the produced droplets.

\section{Droplet Flow Rates}

The balance of specific energy rates in the atomization can be obtained by taking the time derivative of Eq. (1): 


$$
\varepsilon_{\Delta p}=\varepsilon_{c}+\varepsilon_{v} .
$$

Employing the dimensionless analysis and performing algebraic manipulations similar to those undertaken above for droplet diameters, two dimensionless numbers $\mathrm{N}_{l, c v}=\rho_{l}^{2} \gamma_{l} d^{3} \dot{n}_{d} / \mu_{l}^{3}$ and $\mathrm{N}_{l, p c}=\Delta p_{g j}^{3 / 2} /\left(\gamma_{l} \rho_{l}^{1 / 2} \dot{n}_{d}\right)$ determining the droplet flow rates are established. Correspondingly, using the timescales of the involved phenomena we have:

$$
\begin{aligned}
& \mathrm{N}_{l, c v} \sim \frac{\tau_{v}^{3}}{\tau_{c}^{3}}, \\
& \mathrm{~N}_{l, p c} \sim \frac{\tau_{c}^{3}}{\tau_{\Delta p}^{3}} .
\end{aligned}
$$

The energy rate scales and timescales are connected, $\varepsilon_{\Delta p} \sim I_{c}^{2} / \tau_{\Delta p}^{3}, \varepsilon_{c} \sim l_{c}^{2} / \tau_{c}^{3}$ and $\varepsilon_{v} \sim I_{c}^{2} / \tau_{v}^{3}$. As a first approximation, we again assume $I_{c}=d$. Therefore, from Eqs. (10) and (11) we obtain:

$$
\begin{aligned}
& \mathrm{N}_{l, c v} \sim \frac{\varepsilon_{c}}{\varepsilon_{v}}=k_{3} \frac{\varepsilon_{c}}{\varepsilon_{v}}, \\
& \mathrm{~N}_{l, p c} \sim \frac{\varepsilon_{\Delta p}}{\varepsilon_{c}}=k_{4} \frac{\varepsilon_{\Delta p}}{\varepsilon_{c}} .
\end{aligned}
$$

Substituting Eqs. (12) and (13) into Eq. (9), we find:

$$
\mathrm{N}_{l, p c}=k_{3}+\frac{k_{3} k_{4}}{\mathrm{~N}_{l, c v}} \text {. }
$$

The obtained equation connects the rates of specific energies provided by the gas jets and dissipated by the atomized liquid in nondimensional form, and it determines the flow rates of the produced droplets.

\section{Results and Discussion}

The atomization diagrams for diameters and flow rates of water droplets are given in Fig. 2 . The droplet diameter and gas jet pressure were varied in Fig. 2a, and the droplet production rate and droplet diameter were varied at $\Delta p_{g j}=2.5$ bar in Fig. $2 \mathrm{~b}$. The central lines of atomization region on the diagrams were obtained from Eqs. (8) and (14), assuming the proportionality coefficients to be order of unity. The lower and upper boundaries of the atomization region are calculated by assuming tenfold difference of the dimensionless numbers $\mathrm{N}_{d}$ (Fig. 2a) and $\mathrm{N}_{l, p c}$ (Fig. 2b) with respect to those of the corresponding central lines $\mathrm{N}_{d}^{*}=1+\mathrm{La}_{d}^{-1}$ and $\mathrm{N}_{l, p c}^{*}=1+\mathrm{N}_{l, c v}^{-1}$. From Fig. $2 \mathrm{a}$ for $\Delta p_{g j}=2.5$ bar, we can calculate that the atomization process is expected to generate droplets with diameters in the range $40 \mathrm{~nm}-$ $3 \mu \mathrm{m}$, and the mean droplet diameter is $\sim 300 \mathrm{~nm}$. In our experimental studies [2], the arithmetic mean diameter was $\sim 250 \mathrm{~nm}$ and the upper boundary of the obtained number based droplet size distribution was $\sim 3 \mu \mathrm{m}$, whereas the lowest measured droplet diameter was set by the measuring range $100 \mathrm{~nm}-900 \mu \mathrm{m}$ of the utilized laser scattering device (Malvern Spraytec). For the calculated mean diameter of $300 \mathrm{~nm}$, Fig. $2 \mathrm{~b}$ yields $10^{8} \mathrm{drop} / \mathrm{s}$, while $10^{7} \mathrm{drop} / \mathrm{s}$ were measured in the experiment [2]. This difference can be attributed to a theoretical assumption of a continuous atomization process, while in fact there is a periodic disintegration (by a gas 
jet) of bubbles rising to the liquid surface (Fig. 1a). The calculations for other liquids (not shown here), including gasoline, diesel, and solutions of sodium alginate, also demonstrated good agreement between the theory and experiment. Finally, it is worth noting that for the region of $\mathrm{La}_{d} \geq 1$ in Fig. $2 \mathrm{a}$, the distribution of droplet diameters in the atomization region follows a lognormal law, which is widely observed in technical and natural liquid atomization processes [3].

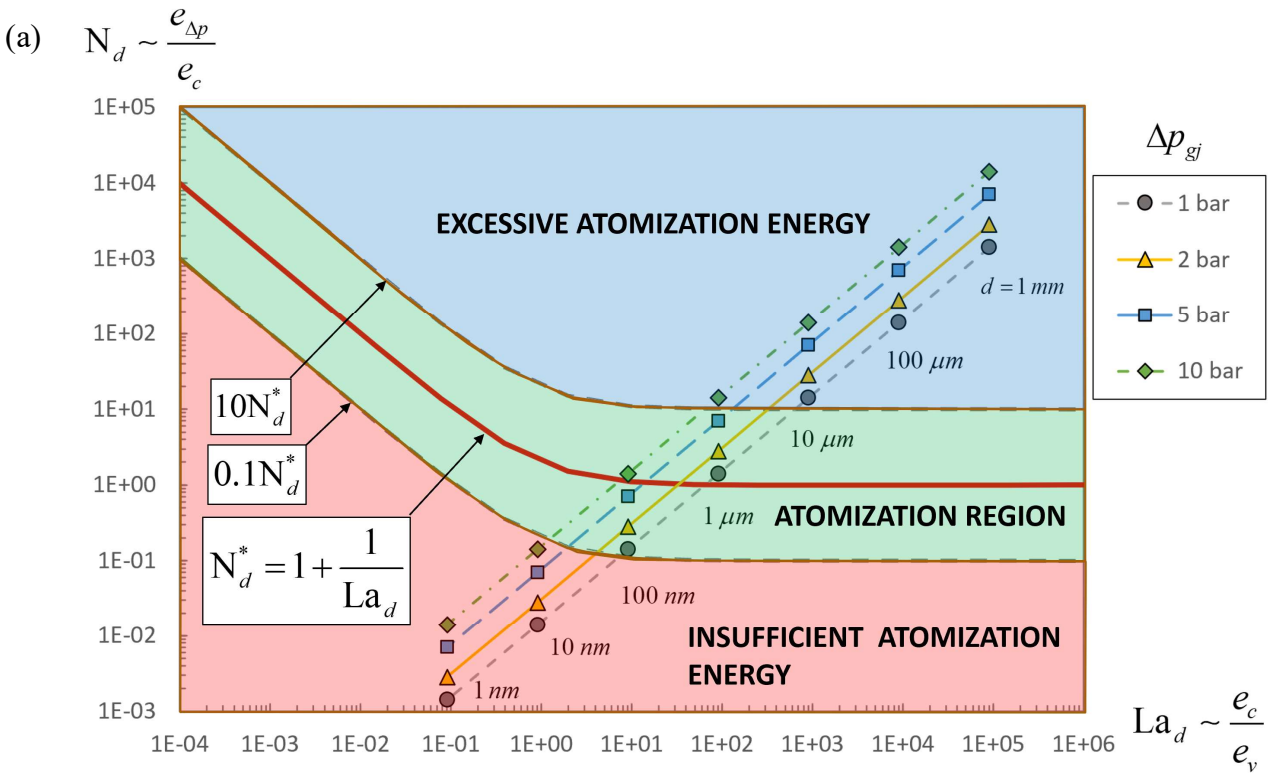

(b) $\mathrm{N}_{l, p c} \sim \frac{\varepsilon_{\Delta p}}{\varepsilon_{c}}$

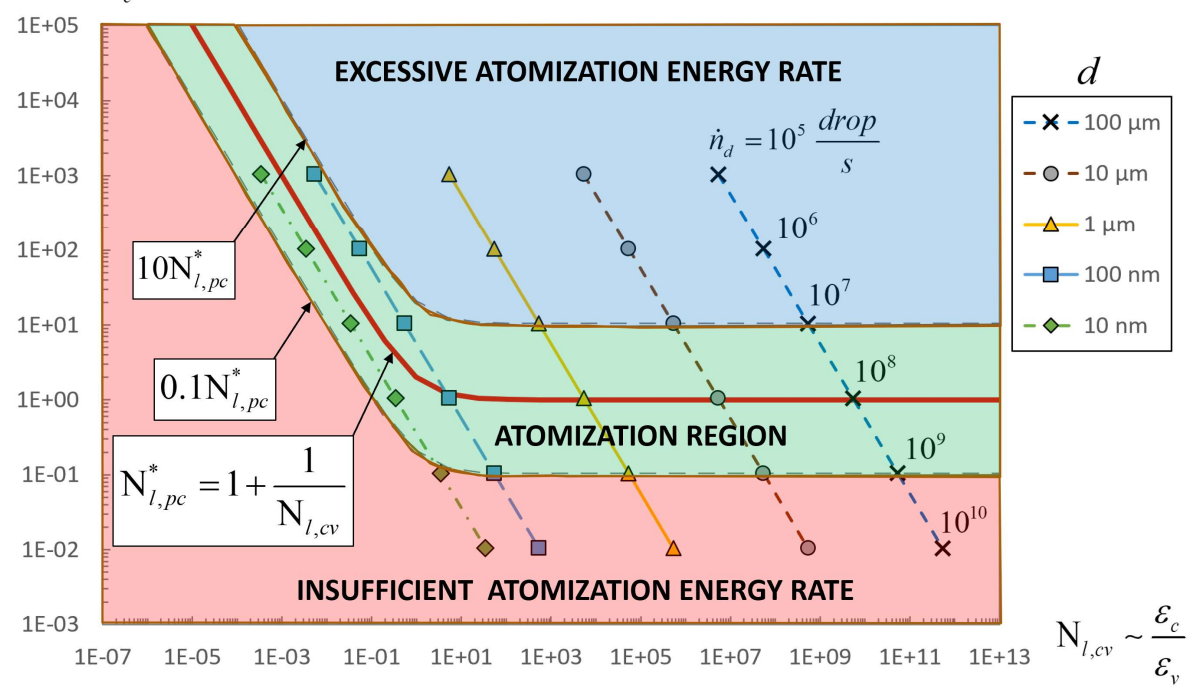

Figure 2. Atomization diagrams for (a) diameters and (b) flow rates of water droplets.

\section{Conclusions}

In this work we demonstrated a new theoretical approach to determine droplet diameters and flow rates in sprays and aerosols using atomization diagrams. The approach is based on the first principles of conservation of mass, energy, and energy rates. The comparison between theoretically predicted and measured droplet diameters and droplet flow rates demonstrated a 
good agreement for the new liquid atomization process generating micro-sprays and aerosols of submicron-diameter droplets.

\section{Acknowledgments}

This work was partially supported by the Foundation for Health Advancement and New Jersey Health Foundation via the Allergan Foundation Innovation Grant Program, Grant \# ALL 01-21.

\section{Nomenclature}

d droplet diameter [m]

$e_{\Delta p} \quad$ specific energy supplied by gas pressure $\left[\mathrm{m}^{2} \mathrm{~s}^{-2}\right]$

$e_{c} \quad$ specific energy stored or dissipated by liquid surface tension $\left[\mathrm{m}^{2} \mathrm{~s}^{-2}\right]$

$e_{v} \quad$ specific energy stored or dissipated by liquid viscosity $\left[\mathrm{m}^{2} \mathrm{~s}^{-2}\right]$

$k \quad$ coefficient of proportionality [-]

$l_{c} \quad$ characteristic length $[\mathrm{m}]$

$\mathrm{La}_{d} \quad$ Laplace number, ratio of energies dissipated by surface tension and viscosity [-]

$\dot{n}_{d} \quad$ production rate of droplets $\left[\mathrm{s}^{-1}\right]$

$\mathrm{N}_{d} \quad$ ratio of energies supplied by gas pressure and dissipated by liquid surface tension [-]

$\mathrm{N}_{l, c v} \quad$ ratio of energy rates dissipated by liquid surface tension and viscosity [-]

$\mathrm{N}_{l, p c} \quad$ ratio of energy rates supplied by gas and dissipated by liquid surface tension [-]

$\gamma_{1} \quad$ surface tension of liquid $\left[\mathrm{N} \mathrm{m}^{-1}\right]$

$\Delta p_{g j} \quad$ differential pressure of gas jet [Pa]

$\varepsilon_{\Delta p} \quad$ specific energy rate supplied by gas pressure $\left[\mathrm{m}^{2} \mathrm{~s}^{-3}\right]$

$\varepsilon_{c} \quad$ specific energy rate stored or dissipated by liquid surface tension $\left[\mathrm{m}^{2} \mathrm{~s}^{-3}\right]$

$\varepsilon_{v} \quad$ specific energy rate stored or dissipated by liquid viscosity $\left[\mathrm{m}^{2} \mathrm{~s}^{-3}\right]$

$\mu_{l} \quad$ dynamic viscosity of liquid [Pa s]

$\rho_{l} \quad$ density of liquid $\left[\mathrm{kg} \mathrm{m}^{-3}\right]$

$\tau_{\Delta p} \quad$ timescale of energy supply to liquid by gas jet [s]

$\tau_{c} \quad$ timescale of capillary Rayleigh breakup of liquid [s]

$\tau_{v} \quad$ timescale of viscous energy dissipation in liquid [s]

\section{References}

[1] Mezhericher, M., Razorenov, N., Mazor, G., Ju, Y., and Stone, H. A., 2019, "Submicron Aerosols of Liquid Fuels: Method of Production, Experimental Characterization and a Semi-Empirical Model," Applied Energy, 235, pp. 1651-1663.

[2] Mezhericher, M., Ladizhensky, I., and Etlin, I., 2017, "Atomization of Liquids by Disintegrating Thin Liquid Films Using Gas Jets," International Journal of Multiphase Flow, 88, pp. 99-115.

[3] Ashgriz, N., ed., 2011, Handbook of Atomization and Sprays: Theory and Applications, Springer, New York, NY. 